\title{
Human equilibrative nucleoside transporter-1 (hENT1) and ribonucleotide reductase regulatory subunit M1 (RRM1) expression; do they have survival impact to pancreatic cancer?
}

\author{
Dae Wook Hwang ${ }^{1, *}$, Eun Shin ${ }^{2, *}$, Jai Young $\mathrm{Cho}^{3}$, Ho-Seong $\mathrm{Han}^{3}$, and Yoo-Seok Yoon ${ }^{3}$ \\ ${ }^{1}$ Division of Hepatobiliary and Pancreatic Surgery, Department of Surgery, Asan Medical Center, University of Ulsan \\ College of Medicine, Seoul, ${ }^{2}$ Department of Pathology, Hallym University Dongtan Sacred Heart Hospital, \\ Hwaseong, ${ }^{3}$ Department of Surgery, Seoul National University College of Medicine, Seoul National University \\ Bundang Hospital, Seongnam, Korea
}

\begin{abstract}
Backgrounds/Aims: Gemcitabine is still one of adjuvant options in chemotherapeutic agent for pancreatic ductal adenocarcinoma (PDAC). Integral membrane transporter protein and intracellular enzymes including human equilibrative nucleoside transporter 1 (hENT1), deoxycytidine kinase (dCK), ribonucleotide reductase (RR) M1, and M2 are known as important factors for chemosensitivity of gemcitabine. We aimed to investigate the correlation between these key molecules and 5-year actual survival in PDAC patients. Methods: The expression of intratumoral hENT1, dCK, RRM1, and RRM2 was assessed immunohistochemically in 160 PDAC patients underwent surgical resection. Association between clininopathologic factors, immunohistochemical results, and overall survival were analyzed. Results: Adjuvant chemotherapy including concurrent chemoradiotherapy was not associated with overall survival (HR, 0.92; 95\% $\mathrm{Cl}$, $0.65-1.31 ; p=0.658)$. High hENT1 expression group did not show statistical survival difference, compared with all others $(\mathrm{HR}, 1.16 ; 95 \% \mathrm{Cl}, 0.82-1.65, p=0.396)$. Gemcitabine therapy and high hENT1 group was compared with all other patients, and no difference in overall survival was identified (HR, 0.99; $95 \% \mathrm{Cl}, 0.68-1.42 ; p=0.940)$. And, gemcitabine therapy and high hENT1 group did not differ statistically from gemcitabine therapy and low hENT1 expression (HR, $0.92 ; 95 \% \mathrm{Cl}, 0.55-1.56 ; p=0.764)$. The intensity of dCK, RRM1, and RRM2 expression was not associated with overall survival $(p=0.413, p=0.138$ and $p=0.061$ ) in univariate analysis. Conclusions: The expression of hENT1, dCK, RRM1 and RRM2 may not be associated with overall survival for patients with pancreatic cancer on gemcitabine adjuvant therapy. These proteins and other factors that may interact with or confound these results should be investigated in the near future. (Ann Hepatobiliary Pancreat Surg 2020;24:127-136)
\end{abstract}

Key Words: Pancreatic ductal adenocarcinoma; Adjuvant chemotherapy; Gemcitabine; hENT1; Overall survival; Surgery

\section{INTRODUCTION}

Despite surgical outcome has improved over the past few decades, pancreatic ductal adenocarcinoma (PDAC) remains one of the most lethal malignancies. After introduction of effective chemotherapeutic agents, the role of chemotherapy in both the adjuvant and neoadjuvant settings, becomes more important than ever. Gemcitabine (2',2'-difluorodeoxycytidine) is a standard chemotherapeutic agent in the treatment of both localized and metastatic PDACs in Korea. However, when administered in un- selected patient populations, gemcitabine only showed a modest benefit in terms of survival. Therefore, better identification of patients who would benefit from administration of gemticibine is required. ${ }^{1}$

As part of efforts to identify predictive biomarkers that forecast the likely response to gemcitabine of PDAC, several studies have investigated the relationship between the chemosensitivity of gemcitabine and integral membrane transporter proteins including human equilibrative nucleoside transporter 1 (hENT1). ${ }^{2,3}$ It was demonstrated that the deficiency in hENT1 was highly associated with the re-

Received: April 20, 2020; Revised: April 20, 2020; Accepted: April 21, 2020

Corresponding author: Yoo-Seok Yoon

Department of Surgery, Seoul National University Bundang Hospital, 166 Gumi-ro, Bundang-gu, Seongnam 13620, Korea

Tel: +82-31-787-7096, Fax: +82-31-787-4078, E-mail: arsyun@gmail.com

*These authors contributed equally to this work.

Copyright (C) 2020 by The Korean Association of Hepato-Biliary-Pancreatic Surgery

This is an Open Access article distributed under the terms of the Creative Commons Attribution Non-Commercial License (http://creativecommons.org/ licenses/by-nc/4.0) which permits unrestricted non-commercial use, distribution, and reproduction in any medium, provided the original work is properly cited. Annals of Hepato-Biliary-Pancreatic Surgery • pISSN: 2508-5778 - elSSN: 2508-5859 
sistant effect of gemcitabine. ${ }^{4,5}$ As a prodrug, intracellular gemcitabine must be phosphorylated by deoxycytidine kinase $(\mathrm{dCK})$ to its mononucleotide in the rate-limiting step of its cellular anabolism. ${ }^{6}$ Gemcitabine monophosphate then converts to its active forms, gemcitabine diphosphate and gemcitabine triphosphate. ${ }^{7}$ The cytotoxicity of gemcitabine is due to its blocking de novo DNA synthesis through inhibition of ribonucleotide reductase, which blocks production of the deoxyribonucleotide precursors required for DNA synthesis. ${ }^{6}$ Ribonucleotide reductase (RR) is a dimeric enzyme composed of a regulatory subunit M1 and a catalytic subunit M2.

While, hENT1, dCK, and RRM1 are important determinants of gemcitabine activity, about the prognostic and predictive values of immunohistochemical assessment of hENT1, dCK, and RRM1 in pancreatic cancer remain unclear, especially in Korean populations. ${ }^{8-10}$ In the present study, we aimed to investigate the correlation between key molecules (hENT1, dCK, RRM1 and RRM2) and 5-year actual survival in patients with PDAC.

\section{MATERIALS AND METHODS}

\section{Study population}

This case-control study was conducted in 200 consecutive and unselected patients with pathologically proven PDAC who underwent curative intent surgery from June 2003 through May 2012 at the Department of Surgery, Seoul National University Bundang Hospital, Korea. Tissue microarrays (TMAs) were made from archived tumor specimen, and 35 patients were excluded because of absence of tumor specimen. After excluding five more patients whose survival status could not be identified as of August 2019, 160 patients were finally enrolled in this study.

\section{Data collection}

Demographic, clinical, and pathological data were collected by an independent reviewer who was masked from the biomarker assessment results. The database was constructed with sex, age at surgery, tumor site, type of operation, tumor stage according to the 8th edition of American Joint Committee on Cancer (AJCC) staging, histologic grade, maximal tumor size, adjuvant therapy, chemotherapeutic regimen, recurrence, disease-free survival, and 5-year actual survival.

\section{Operative procedure}

All type of pancreatectomy were included, such as conventional pancreaticoduodenectomy, pylorus-preserving pancreaticoduodenectomy, distal pancreatectomy, total pancreatectomy, and other types of parenchymal-sparing pancreatectomy. Depends on tumor location, types of pancreatectomy were decided. All patients underwent more than standard lymphadenectomy. When preoperative imaging studies showed invasion into the portal vein, the attending surgeon decided whether to perform portal vein resection. Intraoperative frozen section examination was performed regularly on proximal or distal pancreatic resection margin.

\section{Biomarker assessment}

An independent pathologist masked to clinicopathologic data and survival outcome of patients (E. Shin) reviewed hematoxylin and eosin-stained slides of each patient. Tissue microarrays (TMAs) were made by $2 \mathrm{~mm}$ sized core from formalin-fixed, paraffin-embedded tumor specimens. ${ }^{11}$ Immunohistochemical staining for hENT1 and dCK was performed using Anti-hENT1 rabbit polyclonal antibody (PAB2255, 1:120, Abnova, Taipei, Taiwan) and anti-dCK mouse monoclonal antibody (LS-C172855, 1:50, LSBio, Seattle, WA, USA). ${ }^{12,13}$

RRM1 expression was evaluated with a polyclonal rabbit antibody against human RRM1 (ab81085, 1:100, Abcam, Cambridge, UK) and RRM2 with polyconal goat antibody against human RRM2 (SC-10846, 1:250, Santa Cruz Biotechnology, Dellas, TX, USA). 4- $\mu$ m-thick sections were cut from each of the paraffin tissue blocks, mounted on positively charged slides. Immunohistochemical staining for hENT1, dCK and RRM2 was performed using Leica Bond-max autostainer (Leica Biosystem, Vista, CA). After undergoing heat epitope retrieval for 20 minutes in Epitomic retrieval solution 2 ( $\mathrm{pH} \mathrm{9.0)}$ in the autostainer, the samples were incubated with individual primary antibodies for 15 minutes. Then sides were incubated with polymer for 8 minutes and subsequently treated with DAB substrate for 10 minutes. Treatment with Harris hematoxylin for 1 minute was performed for counterstain. Immunohistochemical staining for RRM2 was manually done. Briefly, antigen was retrieved by treating $\mathrm{pH} 9.0$ Tris-EDTA buffer at $100^{\circ} \mathrm{C}$ for 
20 minutes. Endogenous peroxidase activity was blocked using 3\% hydrogen peroxide for 6 minutes. For protein blocking, slides were incutaed in 5\% normal horse serum for 30 minutes at room temperature. The primary antibody and the antibody binding were detected using an avidin-biotin complex reagent (Vector Lab., Burlingame, CA, USA) for 30 minutes and DAB substrate kit (Vector Lab., Burlingame, CA, USA) for 2 minutes.

The results of immunohistochemical staining was evaluated at $\times 200$ magnificaion under a light microscope. The intensity staining was semi-quantitavely scored as scored as follows: grade 0 , not stained; grade 1, equivocally stained; grade 2, weakly stained; grade 3, moderately stained; and grade 4, stained strongly. Islet cells, plasma and stromal cells were used as internal control. Additionally, the percentage of immune-reactive cells was assessed. For evaluation of intratumoral hENT1 expression, if grade 4 staining was observed in the neoplasms, the sample was considered to have high hENT1 expression, and if grade 0 to 3 staining was observed, the sample was considered to have low hENT1 expression. ${ }^{14}$

\section{Survival}

All patients were followed regularly in outpatient clinics with blood tests or imaging studies, including computed tomography (CT) every 3 to 6 months. Evaluation of recurrence was based on imaging studies. Final survival was assessed in at Aug. 2019 when all patients had achieved more than 5 years of follow-up. Survival information was collected by reviewing electrical medical records or telephone interview. For all patients, postoperative survival time, survival status, and recurrence status were reviewed and recorded.

\section{Statistical analysis}

Statistical analysis was conducted using R 3.5.1. (R Foundation for Statistical Computing, Vienna, Austria). Categorical variables were reported as frequencies, and continuous variables were reported as median with range or mean with standard deviation, as appropriate. Categorical variables or proportions were compared using the chi-square test with continuity correction or Fisher's exact test. Continuous variables were compared using Student's t-test if normally distributed and the Wilcoxon rank-sum test otherwise. Survival curves were obtained using the Kaplan-Meier method. Log rank test and Cox proportional hazards models were used to compare overall survival. Tests of significance were undertaken at the two-tailed alpha level of 0.05 .

\section{RESULTS}

\section{Patients demographics and clinical data}

Of the 160 patients, 93 (58.1\%) were males and 67 $(41.9 \%)$ were females; median age was 65 years (range, 40-89). Pancreatoduodenectomy, distal pancreatectomy, and total pancreatectomy were performed for $96(60.0 \%)$, $54(33.8 \%)$, and $6(3.8 \%)$ patients, respectively. The proportion of patients with preoperative diabetes was significantly different between the two hENT1 intensity groups, with $30(47.6 \%)$ and $28(29.5 \%)$ patients, respectively $(p=0.021)$. Pancreatic neoplasm were confined to the head and body/tail of the pancreas in 98 patients $(62.5 \%)$ and 58 patients $(36.3 \%)$, respectively. One hundred thirty seven patients $(85.6 \%)$ underwent R0 resection.

According to TNM classification, 1 (0.6\%), 3 (1.9\%), and $150(93.8 \%)$ patients had $\mathrm{T} 1, \mathrm{~T} 2$, and $\mathrm{T} 3$ neoplasms, respectively; 97 patients $(60.6 \%)$ had lymph node metastases. No patients had stage IA disease, whereas 3 (1.9\%) patients had stage IB, $58(36.3 \%)$ patients had stage IIA, 92 (57.5\%) patients had stage IIB, and 7 (4.4\%) patients had stage IV disease (Table 1).

Of 160 patients, $93(58.1 \%)$ patients received adjuvant therapy. Among 93 patients, 70 received gemcitabine based adjuvant therapy. The proportion receiving adjuvant chemotherapy was not different between groups, with 46 $(69.7 \%)$ and $47(56.0 \%)$ patients, respectively ( $p=0.121)$. There was no significant difference with gemcitabine therapy (26 (41.3\%) and $44(45.4 \%)$ patients, $p=0.729)$. High intratumoral expression of hENT1 was observed in 97 (60.6\%) patients, and high hENT1 expression with gemcitabine therapy was identified in $44(27.5 \%)$ patients.

DCK and RRM1 expression showed correlation with hENT1 expression, but, there was no significant correlation between RRM2 expression and hENT1 expression (Table 1).

\section{Biomarker assessment and survival}

Table 2 shows the results of analyses of predictive ef- 
Table 1. Comparison of clinicopathologic factors based on the intensity of intratumoral hENT1 expression

\begin{tabular}{|c|c|c|c|c|}
\hline \multirow{2}{*}{ Variables } & \multirow{2}{*}{ Overall } & \multicolumn{2}{|c|}{ hENT1 intensity } & \multirow{2}{*}{$p$-value } \\
\hline & & 0 to $3(n=63)$ & $4(n=97)$ & \\
\hline Age (mean (SD), years) & $64.61(9.8)$ & $66.30(9.1)$ & $63.50(10.1)$ & 0.077 \\
\hline \multicolumn{5}{|l|}{ Sex } \\
\hline Male & $93(58.1)$ & $42(66.7)$ & $51(52.1)$ & 0.126 \\
\hline Female & 67 (41.9) & $21(33.3)$ & $46(47.9)$ & \\
\hline Body Mass Index (mean, $\mathrm{kg} / \mathrm{m}^{2}$ ) & $21.76(3.33)$ & $21.70(3.00)$ & $21.80(3.55)$ & 0.848 \\
\hline \multicolumn{5}{|l|}{ Diabetes, preoperative } \\
\hline Yes & $58(36.7)$ & $30(47.6)$ & $28(29.5)$ & 0.021 \\
\hline No & $100(63.3)$ & $33(52.4)$ & $67(70.5)$ & \\
\hline \multicolumn{5}{|l|}{ Preoperative CA19-9 (U/ml) } \\
\hline$<37$ & $46(29.5)$ & $15(24.6)$ & $31(32.6)$ & 0.322 \\
\hline$\geq 37,<300$ & $56(35.9)$ & $22(36.1)$ & $34(35.8)$ & \\
\hline$\geq 300,<600$ & $18(11.5)$ & $5(8.2)$ & $13(13.7)$ & \\
\hline$\geq 600,<900$ & $7(4.5)$ & $4(6.6)$ & $3(3.2)$ & \\
\hline$\geq 900$ & $29(18.6)$ & $15(24.6)$ & $14(14.7)$ & \\
\hline \multicolumn{5}{|l|}{ Postoperative CA19-9 (U/ml) } \\
\hline$<37$ & $82(54.7)$ & $31(50.0)$ & $51(58.0)$ & 0.732 \\
\hline$\geq 37,<300$ & $49(32.7)$ & $21(33.9)$ & $28(31.8)$ & \\
\hline$\geq 300,<600$ & $9(6.0)$ & $5(8.1)$ & $4(4.5)$ & \\
\hline$\geq 600,<900$ & $3(2.0)$ & $1(1.6)$ & $2(2.3)$ & \\
\hline$\geq 900$ & $7(4.7)$ & $4(6.5)$ & $3(3.4)$ & \\
\hline \multicolumn{5}{|l|}{ Location of tumor } \\
\hline Head & $98(62.0)$ & $40(62.5)$ & $58(60.4)$ & 0.502 \\
\hline Body & $20(12.7)$ & $6(9.4)$ & $14(14.6)$ & \\
\hline Body and tail & $8(5.1)$ & $2(3.1)$ & $6(6.3)$ & \\
\hline Tail & $30(19.0)$ & $14(21.9)$ & $16(16.7)$ & \\
\hline Whole pancreas & $4(2.5)$ & $2(3.1)$ & $2(2.1)$ & \\
\hline \multicolumn{5}{|l|}{ Type of operation } \\
\hline $\begin{array}{l}\text { Pancreaticoduodenectomy including } \\
\text { pylorus-preserving }\end{array}$ & $96(60.0)$ & $37(58.7)$ & $59(60.8)$ & 0.818 \\
\hline Distal pancreatectomy & $54(33.8)$ & $21(33.3)$ & $33(34.0)$ & \\
\hline Total pancreatectomy & $6(3.8)$ & $2(3.2)$ & $4(4.1)$ & \\
\hline Others & $4(2.5)$ & $3(4.8)$ & $1(1.0)$ & \\
\hline \multicolumn{5}{|l|}{$\mathrm{R} 0$ resection } \\
\hline Yes & $137(85.6)$ & $54(84.4)$ & $83(86.5)$ & 0.780 \\
\hline No & $23(14.4)$ & $10(15.6)$ & $13(13.5)$ & \\
\hline \multicolumn{5}{|l|}{$\mathrm{T}$ stage } \\
\hline $\mathrm{T} 1$ & $1(0.6)$ & $0(0.0)$ & $1(1.0)$ & 0.593 \\
\hline $\mathrm{T} 2$ & $3(1.9)$ & $2(3.2)$ & $1(1.0)$ & \\
\hline $\mathrm{T} 3$ & $150(93.8)$ & $58(92.1)$ & $92(94.8)$ & \\
\hline $\mathrm{T} 4$ & $6(3.8)$ & $3(4.8)$ & $3(3.1)$ & \\
\hline \multicolumn{5}{|l|}{$\mathrm{N}$ stage } \\
\hline No & $62(38.8)$ & $20(31.7)$ & $42(43.3)$ & 0.227 \\
\hline N1 & $97(60.6)$ & $43(68.3)$ & $54(55.7)$ & \\
\hline $\mathrm{Nx}$ & $1(0.6)$ & $0(0.0)$ & $1(1.0)$ & \\
\hline \multicolumn{5}{|l|}{$M$ stage } \\
\hline M0 & $155(96.9)$ & $63(100.0)$ & $92(94.8)$ & 0.172 \\
\hline M1 & $5(3.1)$ & $0(0.0)$ & $5(5.2)$ & \\
\hline \multicolumn{5}{|l|}{ Stage, AJCC 8th } \\
\hline IB & $3(1.9)$ & $2(3.2)$ & $1(1.0)$ & 0.154 \\
\hline IIA & $58(36.2)$ & $17(27.0)$ & $41(42.3)$ & \\
\hline IIB & $92(57.5)$ & $42(66.7)$ & $50(51.5)$ & \\
\hline IV & $7(4.4)$ & $2(3.2)$ & $5(5.2)$ & \\
\hline
\end{tabular}


Table 1. Continued

\begin{tabular}{|c|c|c|c|c|}
\hline \multirow{2}{*}{ Variables } & \multirow{2}{*}{ Overall } & \multicolumn{2}{|c|}{ hENT1 intensity } & \multirow{2}{*}{$p$-value } \\
\hline & & 0 to $3(n=63)$ & $4(n=97)$ & \\
\hline \multicolumn{5}{|l|}{ Adjuvant therapy } \\
\hline Yes & $93(58.1)$ & $46(69.7)$ & $47(56.0)$ & 0.121 \\
\hline Chemotherapy, only & 61 & 27 & 34 & \\
\hline CCRT & 32 & 19 & 13 & \\
\hline No & $57(41.9)$ & $20(30.3)$ & $37(44.0)$ & \\
\hline \multicolumn{5}{|c|}{ Gemcitabine adjuvant therapy } \\
\hline Yes & $70(43.8)$ & $26(41.3)$ & $44(45.4)$ & 0.729 \\
\hline No & $90(56.2)$ & $37(58.7)$ & $53(54.6)$ & \\
\hline \multicolumn{5}{|l|}{ hENT1 (intensity) } \\
\hline 0 & $5(3.1)$ & $5(7.9)$ & & \\
\hline 1 & $9(5.6)$ & $9(14.3)$ & & \\
\hline 2 & $10(6.2)$ & $10(15.9)$ & & \\
\hline 3 & $39(24.4)$ & $39(61.9)$ & & \\
\hline 4 & $97(60.6)$ & & $97(100.0)$ & \\
\hline \multicolumn{5}{|l|}{ DCK (intensity) } \\
\hline 0 & $48(30.6)$ & $33(53.2)$ & $15(15.8)$ & $<0.001$ \\
\hline 1 & $0(0.0)$ & $0(0.0)$ & $0(0.0)$ & \\
\hline 2 & $39(24.8)$ & $19(30.6)$ & $20(21.1)$ & \\
\hline 3 & $64(40.8)$ & $10(16.1)$ & $54(56.8)$ & \\
\hline 4 & $6(3.8)$ & $0(0.0)$ & $6(6.3)$ & \\
\hline \multicolumn{5}{|l|}{ RRM1 (intensity) } \\
\hline 0 & $25(16.1)$ & $21(33.3)$ & $4(4.3)$ & $<0.001$ \\
\hline 1 & $15(9.7)$ & $7(11.1)$ & $8(8.7)$ & \\
\hline 2 & $66(42.6)$ & $25(39.7)$ & $41(44.6)$ & \\
\hline 3 & $43(27.7)$ & $8(12.7)$ & $35(38.0)$ & \\
\hline 4 & $6(3.9)$ & $2(3.2)$ & $4(4.3)$ & \\
\hline \multicolumn{5}{|l|}{ RRM2 (intensity) } \\
\hline 0 & $16(10.3)$ & $8(12.7)$ & $8(8.7)$ & 0.150 \\
\hline 1 & $0(0.0)$ & $0(0.0)$ & $0(0.0)$ & \\
\hline 2 & $7(4.5)$ & $1(1.6)$ & $6(6.5)$ & \\
\hline 3 & $62(40.0)$ & $30(47.6)$ & $32(34.8)$ & \\
\hline 4 & $70(45.2)$ & $24(38.1)$ & $46(50.0)$ & \\
\hline hENT1 high+gemcitabine & $44(27.5)$ & $0(0.0)$ & $44(45.4)$ & $<0.001$ \\
\hline Others & $116(72.5)$ & $63(100.0)$ & $53(54.6)$ & \\
\hline
\end{tabular}

hENT1, human equilibrative nucleoside transporter 1; dCK, deoxycytidine kinase; RRM1, ribonucleotide reductase regulatory subunit M1; RRM2, ribonucleotide reductase regulatory subunit M2; AJCC, American Joint Committee on Cancer

fects of various factors including immunohistochemical test results on overall survival. Age, sex, presence of preoperative diabetes and preoperative CA 19-9 level was not associated with overall survival. However, preoperative body mass index (BMI) and postoperative CA 19-9 level over $900 \mathrm{U} / \mathrm{ml}$ displayed significant interactive effects with overall survival (HR, 0.93; 95\% confidence interval (CI), 0.88-0.98; $p=0.007$ and HR, 5.22; 95\% CI, 2.33-11.68; $p<0.001)$. Tumor location, operation type, and extent of resection were not associated with survival. AJCC staging including $\mathrm{T}$ and $\mathrm{N}$ staging shows statisti- cally significant interaction with overall survival.

Adjuvant chemotherapy including concurrent chemoradiotherapy was not associated with overall survival (HR, $0.92 ; 95 \% \mathrm{CI}, 0.65-1.31 ; p=0.658)$. The intensity of hENT1 expression itself was not significantly associated with actual overall survival (Table 2, Fig. 1). After grouping high hENT1 expression and others, there was no statistical survival difference (HR, 1.16; 95\% CI, 0.82-1.65, $p=0.396$ ). To assess interactive effects of gemcitabine therapy, the group with gemcitabine therapy and high hENT1 expression was compared with all other patients, 
Table 2. Univariate overall survival analysis for 160 patients

\begin{tabular}{|c|c|c|c|c|}
\hline \multirow{2}{*}{ Variables } & \multicolumn{4}{|c|}{ Univariate } \\
\hline & \multirow{2}{*}{$\frac{\mathrm{HR}}{1.002}$} & \multicolumn{2}{|c|}{$95 \% \mathrm{CI}$} & \multirow{2}{*}{$\begin{array}{c}p \text {-value } \\
0.848\end{array}$} \\
\hline Age & & 0.984 & 1.020 & \\
\hline Sex & 0.891 & 0.632 & 1.256 & 0.510 \\
\hline BMI & 0.927 & 0.878 & 0.980 & 0.007 \\
\hline Preoperative diabetes & 1.010 & 0.705 & 1.447 & 0.957 \\
\hline Preoperative CA 19-9 (reference, $<37 \mathrm{U} / \mathrm{ml})$ & & & & 0.475 \\
\hline$\geq 37,<300$ & 1.272 & 0.828 & 1.952 & 0.272 \\
\hline$\geq 300,<600$ & 1.183 & 0.657 & 2.130 & 0.576 \\
\hline$\geq 600,<900$ & 1.736 & 0.731 & 4.120 & 0.211 \\
\hline$\geq 900$ & 1.529 & 0.923 & 2.531 & 0.099 \\
\hline Postoperative CA $19-9$ (reference, $<37 \mathrm{U} / \mathrm{ml}$ ) & & & & 0.008 \\
\hline$\geq 37,<300$ & 1.440 & 0.983 & 2.110 & 0.061 \\
\hline$\geq 300,<600$ & 1.568 & 0.751 & 3.272 & 0.231 \\
\hline$\geq 600,<900$ & 1.555 & 0.485 & 4.985 & 0.458 \\
\hline$\geq 900$ & 5.222 & 2.335 & 11.680 & $<0.001$ \\
\hline Location of tumor (reference, head) & & & & 0.884 \\
\hline Body & 0.970 & 0.407 & 2.308 & 0.944 \\
\hline Body and tail & 0.799 & 0.485 & 1.317 & 0.379 \\
\hline Tail & 1.893 & 0.251 & 14.273 & 0.536 \\
\hline Whole pancreas & 0.591 & 0.079 & 4.427 & 0.609 \\
\hline Type of operation (reference, pancreaticoduodenectomy) & & & & 0.235 \\
\hline Distal pancreatectomy & 2.454 & 0.563 & 10.694 & 0.232 \\
\hline Total pancreatectomy & 0.578 & 0.313 & 1.067 & 0.080 \\
\hline Others & 0.389 & 0.113 & 1.345 & 0.136 \\
\hline Extent of resection & 1.013 & 0.531 & 1.934 & 0.968 \\
\hline $\mathrm{T}$ stage & & & & 0.025 \\
\hline $\mathrm{T} 2$ & 0.038 & 0.002 & 0.618 & 0.022 \\
\hline $\mathrm{T} 3$ & 0.159 & 0.021 & 1.174 & 0.071 \\
\hline $\mathrm{T} 4$ & 0.408 & 0.048 & 3.476 & 0.412 \\
\hline $\mathrm{N}$ stage & & & & 0.017 \\
\hline N1 & 1.421 & 1.004 & 2.009 & 0.047 \\
\hline $\mathrm{Nx}$ & 27.920 & 3.388 & 230.051 & 0.002 \\
\hline AJCC 8th stage (reference, stage IB) & & & & 0.032 \\
\hline IIA & 3.668 & 0.506 & 26.581 & 0.198 \\
\hline IIB & 4.690 & 0.651 & 33.764 & 0.125 \\
\hline IV & 9.351 & 1.146 & 76.266 & 0.037 \\
\hline Adjuvant chemotherapy & 0.923 & 0.649 & 1.314 & 0.658 \\
\hline Gemcitabine adjuvant therapy & 1.006 & 0.719 & 1.409 & 0.971 \\
\hline hENT1 (reference, intensity 0) & & & & 0.398 \\
\hline 1 & 0.347 & 0.105 & 1.149 & 0.083 \\
\hline 2 & 0.647 & 0.217 & 1.935 & 0.436 \\
\hline 3 & 0.629 & 0.244 & 1.622 & 0.338 \\
\hline 4 & 0.698 & 0.283 & 1.722 & 0.435 \\
\hline dCK (reference, intensity 0 ) & & & & 0.413 \\
\hline 2 & 1.253 & 0.795 & 1.976 & 0.331 \\
\hline 3 & 0.956 & 0.635 & 1.437 & 0.828 \\
\hline 4 & 0.612 & 0.218 & 1.714 & 0.350 \\
\hline RRM1 (reference, intensity 0) & & & & 0.138 \\
\hline 1 & 0.793 & 0.387 & 1.626 & 0.527 \\
\hline 2 & 1.337 & 0.803 & 2.225 & 0.264 \\
\hline 3 & 0.907 & 0.523 & 1.572 & 0.728 \\
\hline 4 & 0.565 & 0.193 & 1.656 & 0.298 \\
\hline
\end{tabular}


Table 2. Continued

\begin{tabular}{lcccc}
\hline \multirow{2}{*}{ Variables } & \multicolumn{3}{c}{ Univariate } \\
\cline { 2 - 5 } & HR & \multicolumn{2}{c}{$95 \%$ CI } & $p$-value \\
\hline RRM2 (reference, intensity 0) & & & 0.048 \\
2 & 0.475 & 0.155 & 1.458 & 0.193 \\
3 & 0.951 & 0.517 & 1.749 & 0.872 \\
4 & 1.375 & 0.757 & 2.499 & 0.296 \\
hENT1 high & 1.163 & 0.821 & 1.648 & 0.396 \\
hENT1 high+gemcitabine & 0.986 & 0.683 & 1.424 & 0.940 \\
\hline
\end{tabular}

hENT1, human equilibrative nucleoside transporter 1; dCK, deoxycytidine kinase; RRM1, ribonucleotide reductase regulatory subunit M1; RRM2, ribonucleotide reductase regulatory subunit M2; AJCC, American Joint Committee on Cancer
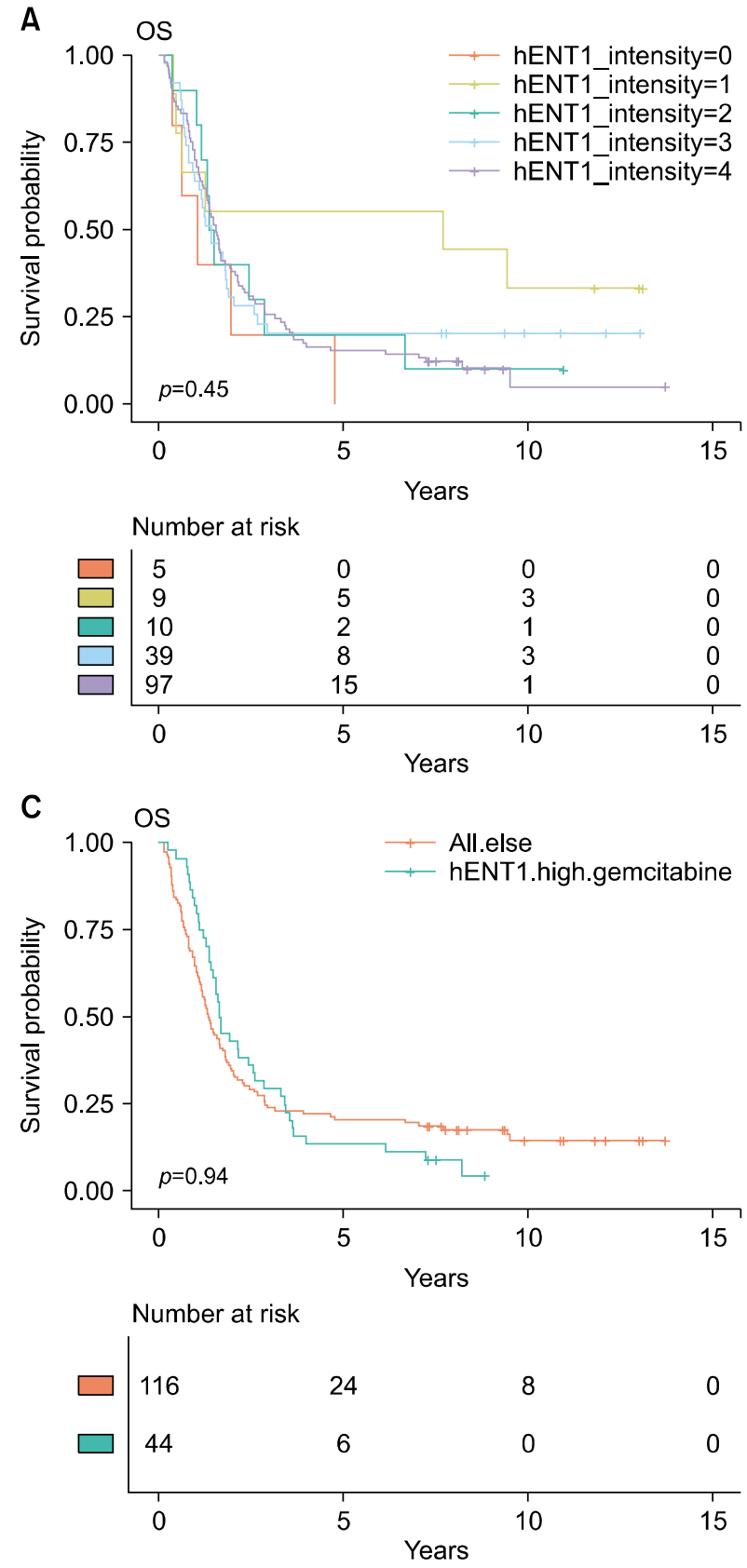

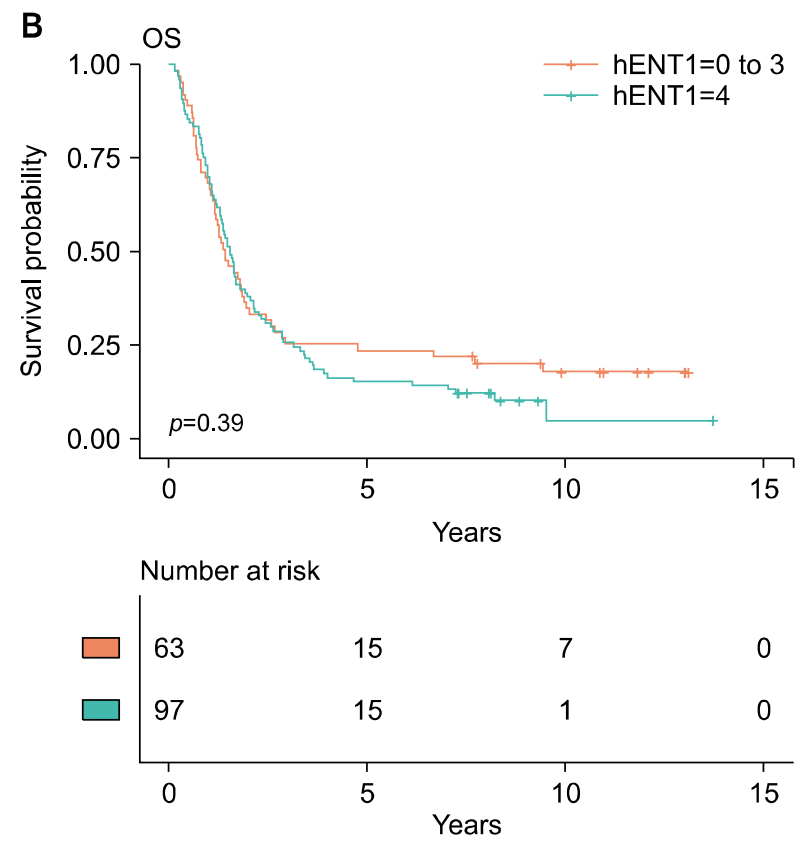

Fig. 1. Kaplan-Meier survival curves. (A) Comparison of overall survival based on intensity of intratumoral hENT1 expression. There was no significant difference in overall survival. (B) High hENT1 expression group was compared with low hENT1 expression group, there was no statistical survival difference (HR, 1.16; 95\% CI, 0.82-1.65, $p=0.396$ ). (C) When the group with gemcitabine therapy and high hENT1 expression was compared with all other patients, and no difference in overall survival was identified (HR, 0.99 ; 95\% CI, 0.68-1.42; $p=0.940$ ). 

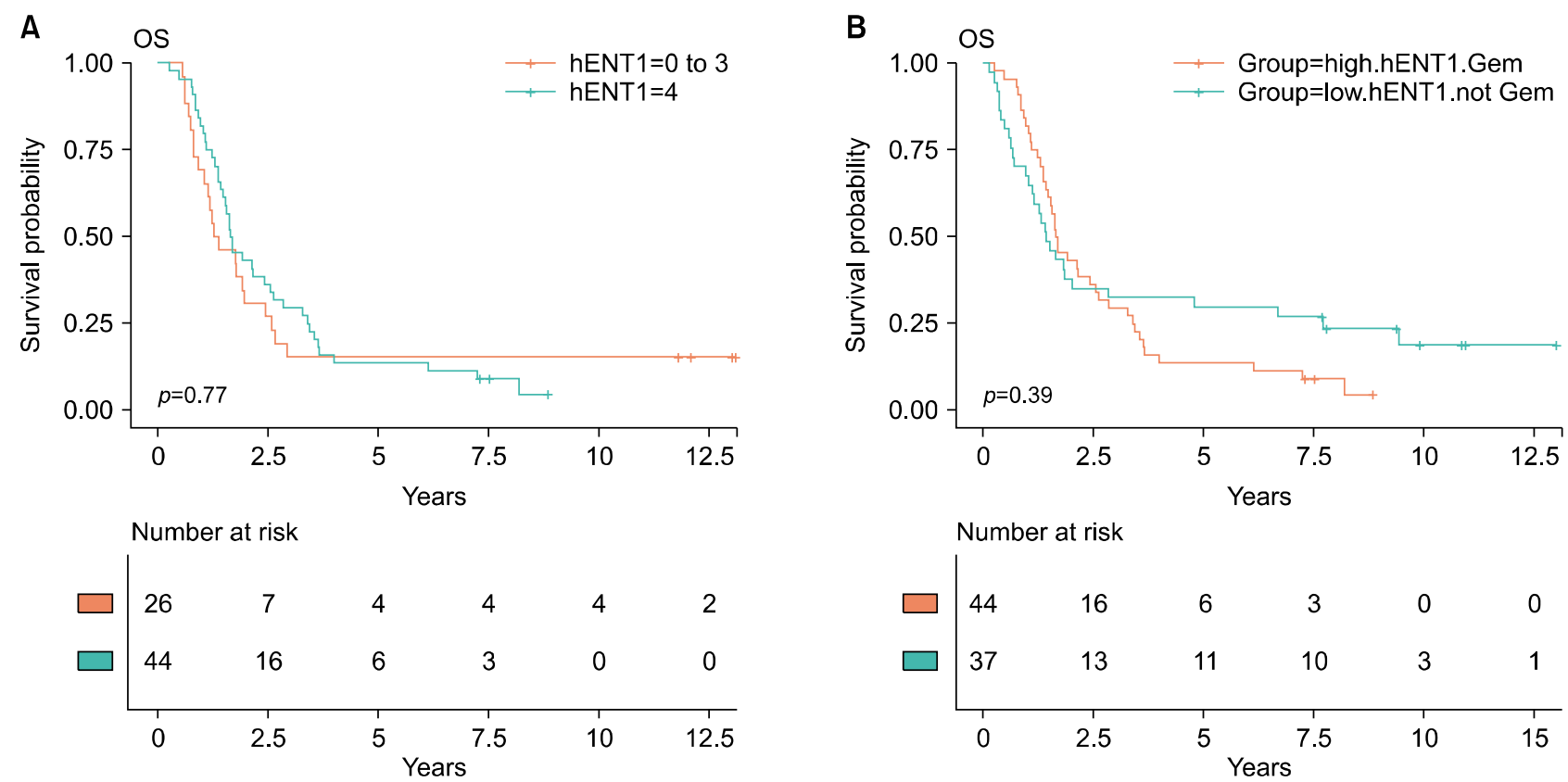

Fig. 2. Kaplan-Meier survival curves. The association between intratumoral hENT1 expression and overall survival according to adjuvant chemotherapeutic agent. (A) Overall survival between the intensity of hENT1 expression within patients with gemcitabine therapy (HR, 0.92; 95\% CI, 0.55-1.56; $p=0.764$ ). (B) The comparison between gemcitabine with high hENT1 expression group and other chemotherapy with low hENT1 did not show significant survival difference (HR, 0.81; 95\% CI, 0.49-1.32; $p=0.394)$.

and no difference in overall survival was identified (HR, $0.99 ; 95 \%$ CI, 0.68-1.42; $p=0.940)$. And, the group with gemcitabine therapy and high hENT1 expression was compared with gemcitabine therapy and low hENT1 expression, there was no statistical survival difference (HR, 0.92; 95\% CI, 0.55-1.56; $p=0.764$ ) (Fig. 2A). The comparison between gemcitabine with high hENT1 expression group and other chemotherapy with low hENT1 did not show significant survival difference (Fig. 2B).

Similarly, the intensity of DCK, RRM1, and RRM2 expression was not associated with overall survival ( $p=0.413$, $p=0.138$ and $p=0.061$ ) in univariate analysis (Table 2).

\section{DISCUSSION}

In this study, we investigated the expression of hENT1, DCK, RRM1 and RRM2 by immunohistochemical analysis to determine their prognostic value in patients with pancreatic cancer. The results showed no significant relationship between protein expression and overall survival. Intratumoral hENT1 expression itself was not a prognostic factor for survival, and high hENT1 expression with gemcitabine therapy was not associated with survival after pancreatic cancer surgery. Moreover, expression of DCK, RRM1, and RRM2 had no correlation with survival.

Although combination chemotherapy with fluorouracil, leucovorin, irinotecan, and oxaliplatin (FOLFIRINOX) leads to longer overall survival than gemcitabine therapy, gemcitabine still plays an important role in systemic chemotherapy for pancreatic cancer. ${ }^{15}$ Survival rate improves significantly with FOLFIRINOX, but gemcitabine remains an option because of the high incidence of toxic effects with FORFIRINOX. There are many reports about adjuvant gemcitabine therapy and prognostic factors. The majority of these reports showed that gatekeepers for intracellular uptake of gemcitabine (hENT1) and metabolic regulators of gemcitabine after intracellular entry (dCK, RRM1, and RRM2) are strongly associated with diseasefree and overall survival in pancreatic cancer. ${ }^{6,14,16-18}$

Our results are not in line with the results of previous reports investigating the prognostic and predictive value of these proteins in pancreatic cancer. First, we analyzed the prognostic value of hENT1 itself, whether or not gemcitabine was used because most previous studies reported an association between hENT1 and survival without analyzing the survival impact of hENT1 expression alone. 
The intensity of hENT1 expression did not show any correlation with survival, even in the high hENT1 expression group ( $p=0.398)$. We then investigated whether survival impact was enhanced when gemcitabine was used in patients with high hENT1. Survival of the group with high hENT1 and gemcitabine was not significantly different, compared with all other patients (HR, 0.986; 95\% CI, $0.68-1.42 ; p=0.940)$. And, even in patients with gemcitabine adjuvant therapy, the survival of the group with high hENT1 did not show the statistical survival difference, compared with low hENT1 (HR, 0.92; 95\% CI, 0.55-1.56; $p=0.764$ ). Expression of dCK also did not show any association with survival, unlike in previous studies. ${ }^{6,14,17}$ According to known intracellular mechanism and previous reports, RRM1 and RRM2 show reverse correlation with the effect of hENT1. However, in our results, RRM1 and RRM2 were not associated with overall survival. Although RRM2 showed marginal significance with survival by Cox proportional hazard ratio, the association was not proven by Kaplan-Meier survival curve.

This study may have had different results than the majority of previous studies for two main reasons. The first is the actual length of follow-up. If follow-up is less than 5 years, then 5 -year overall survival may be overestimated compared to actual survival. The second consideration is the size of the study population. Except for a few large scale studies, most previous studies were conducted with small study populations, which affects the association and interpretation of results. ${ }^{6,17-19}$

Our study had several limitations. First, this is a case-control study. A nonrandomized observational study should be interpreted with caution because of the potential for bias, especially selection bias. Second, a long study period would affect the outcome. A 10 year study period might coincide with major improvements in surgery, oncology, and general supportive care, which could mask the survival impact of gemcitabine related proteins. Third, inter-rater reliability was not measured. Measurement and selection biases should be avoided using all available methods.

Despite its limitations, this study suggests that expression of hENT1, dCK, RRM1 and RRM2 may not be associated with overall survival for patients with pancreatic cancer on gemcitabine adjuvant therapy. These proteins and other factors that may interact with or confound these results should be investigated in the near future. A well-designed cohort study with expression profiling of all available genes is required to create criteria regarding chemotherapeutic applications in patients with PDAC.

\section{ACKNOWLEDGEMENTS}

This research was supported by Seoul National University Bundang Hospital Research Fund (Grant No. 11-2012-004).

\section{CONFLICT OF INTEREST}

The authors declare no competing interests in relation to this study.

\section{ORCID}

Dae Wook Hwang: https://orcid.org/0000-0002-1749-038X

Eun Shin: https://orcid.org/0000-0002-9627-7914

Jai Young Cho: https://orcid.org/0000-0002-1376-956X

Ho-Seong Han: https://orcid.org/0000-0001-9659-1260

Yoo-Seok Yoon: https://orcid.org/0000-0001-7621-8557

\section{AUTHOR CONTRIBUTIONS}

Conceptualization: DWH, YSY, JYC, HSH. Data curation: DWH, YSY, ES. Formal analysis: DWH, YSY. Methodology: DWH, JYC, HSH, ES. Project administration: DWH, YSY. Visualization: DWH, JYC, HSH. Writing - original draft: DWH, ES. Writing - review \& editing: DWH, YSY.

\section{REFERENCES}

1. Neoptolemos JP, Stocken DD, Bassi C, Ghaneh P, Cunningham D, Goldstein D, et al. Adjuvant chemotherapy with fluorouracil plus folinic acid vs gemcitabine following pancreatic cancer resection: a randomized controlled trial. JAMA 2010;304:10731081.

2. Ritzel MW, Ng AM, Yao SY, Graham K, Loewen SK, Smith $\mathrm{KM}$, et al. Recent molecular advances in studies of the concentrative $\mathrm{Na}+$-dependent nucleoside transporter (CNT) family: identification and characterization of novel human and mouse proteins (hCNT3 and mCNT3) broadly selective for purine and pyrimidine nucleosides (system cib). Mol Membr Biol 2001; 18:65-72.

3. Mori R, Ishikawa T, Ichikawa Y, Taniguchi K, Matsuyama R, Ueda $\mathrm{M}$, et al. Human equilibrative nucleoside transporter 1 is 
associated with the chemosensitivity of gemcitabine in human pancreatic adenocarcinoma and biliary tract carcinoma cells. Oncol Rep 2007;17:1201-1205.

4. García-Manteiga J, Molina-Arcas M, Casado FJ, Mazo A, Pastor-Anglada M. Nucleoside transporter profiles in human pancreatic cancer cells: role of hCNT1 in 2',2'-difluorodeoxycytidineinduced cytotoxicity. Clin Cancer Res 2003;9:5000-5008.

5. Mackey JR, Mani RS, Selner M, Mowles D, Young JD, Belt $\mathrm{JA}$, et al. Functional nucleoside transporters are required for gemcitabine influx and manifestation of toxicity in cancer cell lines. Cancer Res 1998;58:4349-4357.

6. Maréchal R, Bachet JB, Mackey JR, Dalban C, Demetter P, Graham K, et al. Levels of gemcitabine transport and metabolism proteins predict survival times of patients treated with gemcitabine for pancreatic adenocarcinoma. Gastroenterology 2012;143: 664-674.e6.

7. Ruiz van Haperen VW, Veerman G, Vermorken JB, Peters GJ. 2',2'-Difluoro-deoxycytidine (gemcitabine) incorporation into RNA and DNA of tumour cell lines. Biochem Pharmacol 1993; 46:762-766.

8. Sebastiani V, Ricci F, Rubio-Viqueira B, Kulesza P, Yeo CJ, Hidalgo $\mathrm{M}$, et al. Immunohistochemical and genetic evaluation of deoxycytidine kinase in pancreatic cancer: relationship to molecular mechanisms of gemcitabine resistance and survival. Clin Cancer Res 2006;12:2492-2497.

9. Bepler G, Kusmartseva I, Sharma S, Gautam A, Cantor A, Sharma A, et al. RRM1 modulated in vitro and in vivo efficacy of gemcitabine and platinum in non-small-cell lung cancer. J Clin Oncol 2006;24:4731-4737.

10. Nakahira S, Nakamori S, Tsujie M, Takahashi Y, Okami J, Yoshioka S, et al. Involvement of ribonucleotide reductase M1 subunit overexpression in gemcitabine resistance of human pancreatic cancer. Int J Cancer 2007;120:1355-1363.

11. Lee HS, Kim WH. Tissue array methods for high-throughput clinicopathologic research. Cancer Res Treat 2006;38:1-6.

12. Farrell JJ, Elsaleh H, Garcia M, Lai R, Ammar A, Regine WF, et al. Human equilibrative nucleoside transporter 1 levels predict response to gemcitabine in patients with pancreatic cancer. Gastroenterology 2009;136:187-195.

13. Maréchal R, Mackey JR, Lai R, Demetter P, Peeters M, Polus $\mathrm{M}$, et al. Deoxycitidine kinase is associated with prolonged survival after adjuvant gemcitabine for resected pancreatic adenocarcinoma. Cancer 2010;116:5200-5206.

14. Nakagawa N, Murakami Y, Uemura K, Sudo T, Hashimoto Y, Kondo N, et al. Combined analysis of intratumoral human equilibrative nucleoside transporter 1 (hENT1) and ribonucleotide reductase regulatory subunit M1 (RRM1) expression is a powerful predictor of survival in patients with pancreatic carcinoma treated with adjuvant gemcitabine-based chemotherapy after operative resection. Surgery 2013;153:565-575.

15. Conroy T, Hammel P, Hebbar M, Ben Abdelghani M, Wei AC, Raoul JL, et al. FOLFIRINOX or gemcitabine as adjuvant therapy for pancreatic cancer. N Engl J Med 2018;379:2395-2406.

16. Morinaga S, Nakamura $\mathrm{Y}$, Watanabe T, Mikayama H, Tamagawa $\mathrm{H}$, Yamamoto $\mathrm{N}$, et al. Immunohistochemical analysis of human equilibrative nucleoside transporter-1 (hENT1) predicts survival in resected pancreatic cancer patients treated with adjuvant gemcitabine monotherapy. Ann Surg Oncol 2012;19 Suppl 3:S558S564.

17. Sierzega M, Pach R, Kulig P, Legutko J, Kulig J. Prognostic implications of expression profiling for gemcitabine-related genes (hENT1, dCK, RRM1, RRM2) in patients with resectable pancreatic adenocarcinoma receiving adjuvant chemotherapy. Pancreas 2017;46:684-689.

18. Farrell JJ, Moughan J, Wong JL, Regine WF, Schaefer P, Benson $\mathrm{AB}$ 3rd, et al. Precision medicine and pancreatic cancer: a gemcitabine pathway approach. Pancreas 2016;45:1485-1493.

19. Elander NO, Aughton K, Ghaneh P, Neoptolemos JP, Palmer $\mathrm{DH}$, Cox TF, et al. Expression of dihydropyrimidine dehydrogenase (DPD) and hENT1 predicts survival in pancreatic cancer. Br J Cancer 2018;118:947-954. 